\title{
Multiple positive solutions of second-order nonlinear difference equations with discrete singular $\phi$-Laplacian
}

Xiaoxiao Su${ }^{1}$ and Ruyun $\mathrm{Ma}^{1^{*}}$

\section{"Correspondence:}

mary@nwnu.edu.cn

${ }^{1}$ Department of Mathematics,

Northwest Normal University,

Lanzhou 730070, P.R. China

\section{Springer}

\begin{abstract}
We consider the existence and multiplicity of positive solutions of the Dirichlet problem for the quasilinear difference equation

$$
\left\{\begin{array}{l}
-\nabla[\phi(\Delta u(t))]=\lambda a(t, u(t))+\mu b(t, u(t)), \quad t \in \mathbb{T}, \\
u(1)=u(N)=0,
\end{array}\right.
$$

where $\lambda, \mu \geq 0, \mathbb{T}=\{2, \ldots, N-1\}$ with $N>3, \phi(s)=s / \sqrt{1-s^{2}}$. The function $f:=\lambda a(t, s)+\mu b(t, s)$ is either sublinear, or superlinear, or sub-superlinear near $s=0$. Applying the topological method, we prove the existence of either one or two, or three positive solutions.
\end{abstract}

MSC: 39A10; 39A27; 47H11

Keywords: Discrete $\phi$-Laplacian; Positive solutions; Multiplicity; Topological method

\section{Introduction}

Let $\mathbb{Z}$ denote the integer set for $N \in \mathbb{Z}$ with $N>3, \mathbb{T}:=\{2, \ldots, N-1\}, \hat{\mathbb{T}}:=\{1, \ldots, N\}$. In this paper, we are concerned with existence and multiplicity of positive solutions for the quasilinear difference boundary value problem

$$
\left\{\begin{array}{l}
-\nabla[\phi(\Delta u(t))]=\lambda a(t, u(t))+\mu b(t, u(t)), \quad t \in \mathbb{T}, \\
u(1)=u(N)=0,
\end{array}\right.
$$

where $\lambda, \mu \geq 0, \Delta u(t)=u(t+1)-u(t)$ is the forward difference operator, $\nabla u(t)=u(t)-$ $u(t-1)$ is the backward difference operator, $\phi:(-1,1) \rightarrow \mathbb{R}$ is given by $\phi(s)=s / \sqrt{1-s^{2}}$, $a, b: \mathbb{T} \times \mathbb{R} \rightarrow \mathbb{R}$ are continuous functions.

Recently, Ma, Wei, and Chen [12] studied the existence and multiplicity of positive solutions for the quasilinear two-point boundary value problem

$$
\left\{\begin{array}{l}
-\left(\frac{u^{\prime}}{\sqrt{1-\left|u^{\prime}\right|^{2}}}\right)^{\prime}=\lambda u^{q}+\mu u^{p}, \quad t \in[0, L) \\
u^{\prime}(0)=u(L)=0 .
\end{array}\right.
$$

(c) The Author(s) 2020. This article is licensed under a Creative Commons Attribution 4.0 International License, which permits use, sharing, adaptation, distribution and reproduction in any medium or format, as long as you give appropriate credit to the original author(s) and the source, provide a link to the Creative Commons licence, and indicate if changes were made. The images or other third party material in this article are included in the article's Creative Commons licence, unless indicated otherwise in a credit line to the material. If material is not included in the article's Creative Commons licence and your intended use is not permitted by statutory regulation or exceeds the permitted use, you will need to obtain permission directly from the copyright holder. To view a copy of this licence, visit http://creativecommons.org/licenses/by/4.0/. 
Here, $0<q<1<p$. They showed that there exist a constant $\mu^{*}>0$ and a function $\Lambda^{*}(\cdot)>0$ such that, for every $\mu>\mu^{*}$ and all $\lambda \in\left(0, \Lambda^{*}(\mu)\right),(1.2)$ has at least three positive solutions. We note that (1.2) is the special case of a one-dimensional version of the Dirichlet problem associated with the Minkowski-curvature equation

$$
\begin{cases}-\operatorname{div}\left(\frac{\nabla u}{\sqrt{1-|\nabla u|^{2}}}\right)=\lambda f(x, u)+\mu g(x, u), & \text { in } \Omega, \\ u=0, & \text { on } \partial \Omega\end{cases}
$$

where $\Omega \subset \mathbb{R}^{N}$. As it is well known, (1.3) plays an important role in differential geometry and in the theory of relativity. In 2012, Corsato et al. [6] studied the multiplicity of positive solutions of (1.3) with $N=1$. For (1.2), [6, Theorem 2.4] is reduced to the following.

Theorem A Let $0<q<1<p$. Then there exist $\mu^{*}>0$ and a function $\lambda(\cdot):\left(\mu^{*},+\infty\right) \rightarrow$ $(0, \infty)$ such that, for all $\mu>\mu^{*}$ and all $\lambda \in(0, \lambda(\mu))$, problem (1.2) has at least three positive solutions.

Theorem A has been extended by Corsato et al. [7] to the problem in general domain $\Omega$, see [7, Theorem 3.1(iv)].

The existence of solutions for quasilinear difference equations subject to diverse boundary conditions has been investigated by several authors, we refer the reader to [1$5,11,13,14]$ and the references therein. However, very little is known about the multiplicity of positive solutions for the discrete analog of Dirichlet problem (1.3). Motivated by the interesting studies above and the references therein, we have a natural question whether or not the similar results are still true for discrete problem (1.1). As we all know, some basic ideas from differential calculus are not necessarily available in the field of difference equations, such as mean value theorem and Poincaré inequality. Thus, new challenges are faced and innovation is required.

The rest paper is arranged as follows: in Sect. 2, we state some notations and preliminary results. Finally, in Sect. 3, we state and prove our main results.

\section{Preliminary results}

For $\mathbf{u}=(u(1), \ldots, u(N)) \in \mathbb{R}^{N}$, set $|\mathbf{u}|_{\infty}=\max _{1 \leq t \leq N}|u(t)|$, If $\mathbf{a}, \mathbf{b} \in \mathbb{R}^{N}$, we write $\mathbf{a} \leq \mathbf{b}$ if $a(t) \leq b(t)$ for every $1 \leq t \leq N$. If $\mathbf{a} \leq \mathbf{b}$ and there exists $t_{0} \in\{1, \ldots, N\}$ such that $a\left(t_{0}\right)<$ $b\left(t_{0}\right)$, then we write $\mathbf{a}<\mathbf{b}$. If $a(t)<b(t)$ for every $1 \leq t \leq N$, we write $\mathbf{a} \ll \mathbf{b}$.

For any $\mathbf{u}=(u(1), \ldots, u(N)) \in \mathbb{R}^{N}$, we define

$$
\Delta \mathbf{u}=(\Delta u(1), \ldots, \Delta u(N-1)) \in \mathbb{R}^{N-1},
$$

where $\Delta u(t)$ is defined by

$$
\Delta u(t)=u(t+1)-u(t), \quad 1 \leq t \leq N-1 .
$$

If $|\Delta \mathbf{u}|_{\infty}<1$, then we define

$$
\nabla[\phi(\Delta \mathbf{u})]=(\nabla[\phi(\Delta u(2))], \ldots, \nabla[\phi(\Delta u(N-1))]) \in \mathbb{R}^{N-2},
$$


where $\nabla[\phi(\Delta u(t))]$ is defined by

$$
\nabla[\phi(\Delta u(t))]=\phi(\Delta u(t))-\phi(\Delta u(t-1)), \quad 2 \leq t \leq N-2 .
$$

For any $m, n \in \mathbb{R}$, let $B=\{m+1, \ldots, n-1\}, \partial B=\{m, n\}$, then $\hat{B}:=\{m, \ldots, n\}$.

Let

$$
Y=\left\{\mathbf{u} \in \mathbb{R}^{N}: u(1)=u(N)=0\right\}
$$

with the norm $|\mathbf{u}|_{\infty}:=\max _{1 \leq j \leq N}|u(j)|$.

A solution of problem (1.1) is a vector $\mathbf{u} \in \mathbb{R}^{N}$ satisfying (1.1) and such that $|\triangle \mathbf{u}|_{\infty}<1$. A nontrivial solution of problem (1.1) is a solution of (1.1) such that $\mathbf{u} \neq \mathbf{0}$. A positive solution of problem (1.1) is a solution of (1.1) such that $\mathbf{u}>\mathbf{0}$. Further, it is said to be strictly positive solution if $\mathbf{u} \gg \mathbf{0}$.

Lemma 2.1 Suppose that $\mathbf{v}_{1}, \mathbf{v}_{2} \in \mathbb{R}^{N}$ satisfy $\mathbf{v}_{1} \leq \mathbf{v}_{2}$. Let $\mathbf{u}_{\mathbf{i}} \in \mathbb{R}^{N}$ be such that $\left|\triangle \mathbf{u}_{\mathbf{i}}\right|_{\infty}<1$ and

$$
-\nabla\left[\phi\left(\Delta u_{i}(t)\right)\right]=v_{i}(t), \quad i=1,2, t \in \mathbb{T} .
$$

Then

$$
u_{2}(t) \leq u_{1}(t)-\min _{t \in\{1, N\}}\left(u_{1}(t)-u_{2}(t)\right) .
$$

Proof Fix $\mathbf{v} \in \mathbb{R}^{N}$ and suppose that $\mathbf{u} \in \mathbb{R}^{N}$ is such that $|\Delta \mathbf{u}|_{\infty}<1$ and

$$
-\nabla[\phi(\Delta u(t))]=v(t), \quad t \in \mathbb{T} .
$$

Let us set

$$
C_{u}=\left\{\mathbf{z} \in \mathbb{R}^{N}:|\triangle \mathbf{z}|_{\infty} \leq 1, t \in \mathbb{T} \text { and } z(1)=u(1), z(N)=u(N)\right\}
$$

and define the functional $T_{\nu}: C_{u} \rightarrow \mathbb{R}$ by

$$
T_{\nu}(\mathbf{w})=\sum_{t=2}^{N-1} \sqrt{1-|w(t)|^{2}}+\sum_{t=2}^{N-1} v(t) w(t)
$$

for all $\mathbf{w} \in C_{u}$. We claim that $\mathbf{u}$ maximizes $T_{v}$ in $C_{u}$. Indeed, pick any $\mathbf{z} \in C_{u}$ : multiplying (2.1) by $u-z$ and summing as $t$ goes from 2 to $N-1$, we get

$$
\begin{aligned}
& \sum_{t=2}^{N-1}-\nabla[\phi(\Delta u(t))](u(t)-z(t))=\sum_{t=2}^{N-1} v(t)(u(t)-z(t)), \\
& \sum_{t=2}^{N-1} \phi(\Delta u(t)) \triangle(u(t)-z(t))=\sum_{t=2}^{N-1} v(t)(u(t)-z(t)) .
\end{aligned}
$$


By the concavity of the function $y \mapsto \sqrt{1-y^{2}}$, we obtain

$$
\sum_{t=2}^{N-1} \sqrt{1-|\triangle z|^{2}}-\sum_{t=2}^{N-1} \sqrt{1-|\triangle u|^{2}} \leq \sum_{t=2}^{N-1} \phi(\Delta u(t)) \triangle(u(t)-z(t))
$$

Combining (2.2) and (2.3) yields $T_{v}(\mathbf{z}) \leq T_{v}(\mathbf{u})$, i.e., $\mathbf{u}$ is a maximizer of $T_{v}$ in $C_{u}$. Accordingly, we have that $\mathbf{u}_{1}$ and $\mathbf{u}_{2}$ are maximizers of $T_{v_{1}}$ in $C_{u_{1}}$ and of $T_{v_{2}}$ in $C_{u_{2}}$, respectively.

Let $C=\min _{t \in\{1, N\}}\left(u_{1}(t)-u_{2}(t)\right)$, choose $\varepsilon>0$ and define $\tilde{u}_{1}=u_{1}-C+\varepsilon$ and $\Omega^{+}=\{t \in$ $\left.\mathbb{T}: u_{2}(t)>\tilde{u}_{1}(t)\right\}$. Assume that $\Omega^{+}$is nonempty. Then, by uniqueness of the solution of this variational problem, $T_{v_{2}}\left(\tilde{\mathbf{u}}_{1}\right) \leq T_{v_{2}}\left(\mathbf{u}_{2}\right)$, and hence

$$
\begin{aligned}
\sum_{t=2}^{N-1} \sqrt{1-\left|\triangle \tilde{u}_{1}\right|^{2}} & \leq \sum_{t=2}^{N-1}\left(\sqrt{1-\left|\triangle u_{2}\right|^{2}}+\left(\tilde{u}_{1}-u_{2}\right) v_{2}\right) \\
& =\sum_{t=2}^{N-1}\left(\sqrt{1-\left|\triangle u_{2}\right|^{2}}+\left(\tilde{u}_{1}-u_{2}\right) v_{1}+\left(\tilde{u}_{1}-u_{2}\right)\left(v_{2}-v_{1}\right)\right)
\end{aligned}
$$

Thus, since $\tilde{u}_{1}<u_{2}$ on $\Omega^{+}$by construction and $\mathbf{v}_{1} \leq \mathbf{v}_{2}, t \in \mathbb{T}$, this becomes

$$
\sum_{t=2}^{N-1}\left(\sqrt{1-\left|\triangle \tilde{u}_{1}\right|^{2}}-\tilde{u}_{1} v_{1}\right) \leq \sum_{t=2}^{N-1}\left(\sqrt{1-\left|\triangle u_{2}\right|^{2}}-u_{2} v_{1}\right)
$$

which contradicts the maximality of $T_{\nu_{1}}\left(\tilde{\mathbf{u}}_{1}\right)$. Hence $\Omega^{+}$is empty, and since $\varepsilon>0$ was arbitrary, the result follows.

Lemma 2.2 (Difference mean value theorem, see [10]) Let $u(t)$ be defined on $\{a, \ldots, b\}$, then there exists $c \in\{a+1, \ldots, b-1\}$ such that one of the following holds:

(i) $\triangle u(c) \leq \frac{u(a)-u(b)}{b-a} \leq \Delta u(c-1)$;

(ii) $\triangle u(c) \geq \frac{u(a)-u(b)}{b-a} \geq \Delta u(c-1)$.

Lemma 2.3 Suppose that $\mathbf{v} \in \mathbb{R}^{N}$, and let $\mathbf{u}$ be a solution of

$$
\left\{\begin{array}{l}
-\nabla[\phi(\Delta u(t))]=v(t), \quad t \in \mathbb{T} \\
u(1)=u(N)=0
\end{array}\right.
$$

Then, for any given $\Lambda>0$, there exist constants $\delta \in(0,1)$ and c such that, for every $|\mathbf{v}|_{\infty} \leq \Lambda$, the following estimates hold:

$$
|\triangle \mathbf{u}|_{\infty}<1-\delta, \quad|\mathbf{u}|_{\infty} \leq c|\mathbf{v}|_{\infty}
$$

Proof By Lemma 2.2, let $\tau$ be the maximum point of $u(t)$, then $\Delta u(\tau) \leq \frac{u(N)-u(1)}{N-1}=0 \leq$ $u(\tau-1)$. Summing both sides of (2.4) from $t+1$ to $\tau$ and $\tau-1$, respectively, we have

$$
\triangle u(t) \leq \phi^{-1}\left(\sum_{s=t+1}^{\tau} v(s)\right)
$$


and

$$
\Delta u(t) \geq \phi^{-1}\left(\sum_{s=t+1}^{\tau-1} v(s)\right)
$$

Since $\phi^{-1}:(-\infty, \infty) \rightarrow(-1,1)$ is given by $\phi^{-1}(s)=s / \sqrt{1+s^{2}}, \phi^{-1}$ is increasing on $(-\infty, \infty)$ and it is an odd function, thus

$$
\Delta u(t) \leq \phi^{-1}\left(\sum_{s=t+1}^{\tau} v(s)\right) \leq \phi^{-1}\left(\left|\sum_{s=t+1}^{\tau} v(s)\right|\right) \leq \phi^{-1}\left(\sum_{s=t+1}^{\tau}|v(s)|\right)
$$

and

$$
\begin{aligned}
\Delta u(t) & \geq \phi^{-1}\left(\sum_{s=t+1}^{\tau-1} v(s)\right)=-\phi^{-1}\left(-\sum_{s=t+1}^{\tau-1} v(s)\right) \\
& \geq-\phi^{-1}\left(\left|\sum_{s=t+1}^{\tau-1} v(s)\right|\right) \geq-\phi^{-1}\left(\sum_{s=t+1}^{\tau}|v(s)|\right) .
\end{aligned}
$$

Since $|\mathbf{v}|_{\infty} \leq \Lambda$, there exists a constant $\delta>0$ such that $\phi^{-1}:[0,(\tau-t) \Lambda] \rightarrow[0,1-\delta], t \in \mathbb{T}$, i.e., $|\Delta \mathbf{u}|_{\infty}<1-\delta$. Moreover, by [14, Lemma 2.2, Lemma 2.3], Dirichlet problem (2.4) is equivalent to the problem

$$
\left\{\begin{array}{l}
-\nabla[(\Delta u(t))]=h(\Delta u(t), \Delta u(t-1)) v(t), \quad t \in \mathbb{T} \\
u(1)=u(N)=0
\end{array}\right.
$$

with

$$
h(y, z)= \begin{cases}\frac{\sqrt{1-|y|^{2}} \sqrt{1-|z|^{2}}\left[\sqrt{1-|y|^{2}}+\sqrt{1-|z|^{2}}\right]}{\sqrt{1-|z|^{2}} \sqrt{1-|y|^{2}}+1+z y}, & |y|<1,|z|<1, \\ 0, & |y| \geq 1,|z| \geq 1\end{cases}
$$

and $h(y, z) \leq 2$. Summing both sides of $(2.5)$ from 1 to $N$, it is easy to see that there exists a constant $c$ such that $|\mathbf{u}|_{\infty} \leq c|\mathbf{v}|_{\infty}$.

The strong maximum principle is as follows.

Lemma 2.4 Fix $\mathbf{u} \in Y$ such that either

$$
u(t)>0 \quad \text { or } \quad-\nabla[\phi(\Delta u(t))] \geq 0
$$

for all $t \in \mathbb{T}$. Then, either $\mathbf{u}>\mathbf{0}$ in $\mathbb{T}$ or $\mathbf{u} \equiv 0$.

Proof Fix $\mathbf{u} \in Y$ satisfying (2.6) and such that $\mathbf{u} \not \equiv \mathbf{0}$. From (2.6), if $u(j) \leq 0, j \in \mathbb{T}$, one has

$$
-\nabla[\phi(\Delta u(t))] \geq 0
$$


Now, we claim that $u(2)>0$. Arguing by contradiction, we assume $u(2) \leq 0$. Applying (2.6) with $j=2$, we have

$$
-\nabla[\phi(\Delta u(2))] \geq 0
$$

so

$$
\frac{u(3)-u(2)}{\sqrt{1-(\triangle u(2))^{2}}} \leq \frac{u(2)}{\sqrt{1-(\triangle u(1))^{2}}}
$$

Since $u(2) \leq 0$, we obtain that

$$
\frac{u(3)-u(2)}{\sqrt{1-(\Delta u(2))^{2}}} \leq \frac{u(2)}{\sqrt{1-(\Delta u(1))^{2}}} \leq 0,
$$

we have $u(3) \leq u(2)$. Let $j=3,4, \ldots, N-1$, repeating the same computation, we get

$$
u(N) \leq u(N-1) \leq \cdots \leq u(2) \leq 0 .
$$

Since $u(N)=0$, we have $u \equiv 0$ and this is absurd, so our claim is proved. Moreover we can prove $u(3)>0, \ldots, u(N-1)>0$ and, finally, in the same way, we obtain $u(N)>0$. Hence, the proof is complete.

Lemma 2.5 Let $B, B_{1}$ be bounded sets and $\hat{B}_{1} \subset B$. Let $\mathbf{v} \in \mathbb{R}^{N}$ be such that $v(t)>0, t \in B_{1}$ and suppose that the solution $\mathbf{u}$ of (2.4) satisfies $\mathbf{u} \geq \mathbf{0}$ in $B$. Then $\min _{\hat{B}_{1}} \mathbf{u}>0$.

Proof Since $v(t)>0, t \in B_{1}$, by the strong maximum principle, $u(t)>0, t \in B_{1}$. Suppose that $u\left(t_{0}\right)=0, t_{0} \in \partial B_{1} \subset B$. Since

$$
\triangle u\left(t_{0}\right)=u\left(t_{0}+1\right)-u\left(t_{0}\right)>0, \quad \triangle u\left(t_{0}-1\right)=u\left(t_{0}\right)-u\left(t_{0}-1\right) \leq 0,
$$

thus

$$
\begin{aligned}
-\nabla\left[\phi\left(\Delta u\left(t_{0}\right)\right)\right] & =-\phi\left(\Delta u\left(t_{0}\right)\right)+\phi\left(\Delta u\left(t_{0}-1\right)\right) \\
& =-\frac{\Delta u\left(t_{0}\right)}{\sqrt{1-\left(\Delta u\left(t_{0}\right)\right)^{2}}}+\frac{\Delta u\left(t_{0}-1\right)}{\sqrt{1-\left(\Delta u\left(t_{0}-1\right)\right)^{2}}} \\
& \leq 0,
\end{aligned}
$$

this is in contradiction with the strong maximum principle.

Lemma 2.6 Let us consider the Dirichlet problem

$$
\left\{\begin{array}{l}
-\nabla[\phi(\Delta u(t))]=\gamma u^{p}, \quad t \in \mathbb{T}, \\
u(1)=u(N)=0
\end{array}\right.
$$

where $\gamma>0$ and $p>0$. The following conclusions hold: 
(i) If $p \in(0,1)$, then for every $\gamma>0$ problem (2.7) has at least one solution $\mathbf{u}$ satisfying $\mathbf{u} \gg \mathbf{0}$.

(ii) If $p \geq 1$, then there exists $\gamma^{*}>0$ such that, for every $\gamma>\gamma^{*}$, problem (2.7) has at least one solution $\mathbf{u}$ satisfying $\mathbf{u} \gg \mathbf{0}$.

Proof If $f(t, u(t))=u^{p}, p \in(0,1)$, applying [10, Theorem 8.4.2], when

$$
\lim _{s \rightarrow 0} \frac{f(t, s)}{s}=0
$$

holds, then for every $\gamma>0$, problem (2.7) has at least one solution $\mathbf{u}$ satisfying $\mathbf{u} \gg \mathbf{0}$. If $f(t, u(t))=u^{p}, p \geq 1$, applying [10, Theorem 8.4.3], when

$$
\lim _{s \rightarrow 0} \frac{f(t, s)}{s}=\infty
$$

holds, then there exists $\gamma^{*}>0$ such that, for every $\gamma>\gamma^{*}$, problem (2.7) has at least one solution $\mathbf{u}$ satisfying $\mathbf{u} \gg \mathbf{0}$.

Let us consider the linear eigenvalue problem

$$
\left\{\begin{array}{l}
-\nabla(\Delta u(t))=\lambda m(t) u(t), \quad t \in \mathbb{T} \\
u(1)=u(N)=0
\end{array}\right.
$$

where $\lambda$ is the spectrum parameter and $m(t)>0$. From [9] it follows that there exist $N$ real eigenvalues satisfying

$$
0<\lambda_{1}<\lambda_{2}<\cdots<\lambda_{N}
$$

The following result follows from Rayleigh's inequality of [8].

Lemma 2.7 Let $\lambda_{1}$ be the smallest eigenvalue of (2.8). Then

$$
\lambda_{1} \leq \frac{\sum_{s=1}^{N}[\Delta u(t-1)]^{2}}{\sum_{s=1}^{N} m(t) u^{2}(t)},
$$

where $u(t)$ is any nontrivial real-valued function defined on $\hat{\mathbb{T}}$ with $u(1)=u(N)=0$. Furthermore, equality holds if and only if $u(t)$ is an eigenfunction corresponding to $\lambda_{1}$.

\section{Existence and multiplicity results}

Let us consider problem (1.1). The following assumptions will be considered:

(H) $a, b: \mathbb{T} \times \mathbb{R} \rightarrow \mathbb{R}$ are continuous functions;

(A1) There exist an open set $\mathbb{T}_{1} \subseteq \mathbb{T}, a_{1}>0$, and $p_{1} \in(0,1)$ such that

$$
a_{1} x^{p_{1}} \leq a(t, x), \quad t \in \mathbb{T}_{1}, x \in\left[0, \frac{N-1}{2}\right]
$$

(A2) $0 \leq a(t, 0), t \in \mathbb{T}$; 
(A3) There exist $a_{2}>0$ and $p_{2} \in(0,1)$ such that

$$
a_{2} x^{p_{2}} \geq a(t, x), \quad t \in \mathbb{T}, x \in\left[0, \frac{N-1}{2}\right]
$$

(B1) There exist an open set $\mathbb{T}_{1} \subseteq \mathbb{T}, b_{1}>0$, and $q_{1} \in[1,+\infty)$ such that

$$
b_{1} x^{q_{1}} \leq b(t, x), \quad t \in \mathbb{T}_{1}, x \in\left[0, \frac{N-1}{2}\right]
$$

(B2) $0 \leq b(t, 0), t \in \mathbb{T}$;

(B3) There exist $b_{2}>0$ and $q_{2} \in(1,+\infty)$ such that

$$
b_{2} x^{q 2} \geq b(t, x), \quad t \in \mathbb{T}, x \in\left[0, \frac{N-1}{2}\right] .
$$

Theorem 3.1 Let $(\mathrm{H})$ hold. Then:

(i) If $\mu=0$ and (A1)-(A2) are satisfied, then for every $\lambda>0$ problem (1.1) has at least one positive solution;

(ii) If $\lambda=0$ and (B1)-(B2) are satisfied, then there exists $\mu^{*}>0$ such that, for every $\mu>\mu^{*}$, problem (1.1) has at least one positive solution;

(iii) If $\lambda=0$ and (B1)-(B3) are satisfied, then there exists $\mu^{*}>0$ such that, for every $\mu>\mu^{*}$, problem (1.1) has at least two positive solutions;

(iv) If (A1)-(A3) and (B1)-(B3) are satisfied, then there exist $\mu^{*}>0$ and a function $\lambda(\cdot):\left(\mu^{*},+\infty\right) \rightarrow \mathbb{R}$ such that, for every $\mu>\mu^{*}, \lambda \in(0, \lambda(\mu))$, problem $(1.1)$ has at least three positive solutions.

Proof Step 1. An equivalent problem. Fix $\lambda \geq 0$ and $\mu \geq 0$. Assume (H), (A2) $(\lambda>0)$ and (B2) $(\mu>0)$. Define the functions $\bar{a}, \bar{b}, \bar{f}: \mathbb{T} \times\left[-\frac{N-1}{2}, \frac{N-1}{2}\right] \rightarrow \mathbb{R}$ by

$$
\begin{aligned}
& \bar{a}(t, x)= \begin{cases}a(t, 0), & -\frac{N-1}{2} \leq x<0, \\
a(t, x), & 0 \leq x \leq \frac{N-1}{2},\end{cases} \\
& \bar{b}(t, x)= \begin{cases}b(t, 0), & -\frac{N-1}{2} \leq x<0, \\
b(t, x), & 0 \leq x \leq \frac{N-1}{2},\end{cases}
\end{aligned}
$$

and $\bar{f}=\lambda \bar{a}+\mu \bar{b}$.

Notice that any nontrivial solution $\mathbf{u}$ of the Dirichlet problem

$$
\left\{\begin{array}{l}
-\nabla[\phi(\Delta u(t))]=\lambda \bar{a}(t, u(t))+\mu \bar{b}(t, u(t)), \quad t \in \mathbb{T}, \\
u(1)=u(N)=0
\end{array}\right.
$$


is positive. Indeed, as $\bar{f}(t, x) \geq 0$ for all $t \in \mathbb{T}, x \in\left[-\frac{N-1}{2}, 0\right]$, multiplying the equation in (3.1) by $\mathbf{u}^{-}:=-\min \{\mathbf{u}, \mathbf{0}\}$ and summing by parts, we have

$$
\begin{aligned}
0 & \leq \sum_{t=1}^{N} \bar{f}(t, u) u^{-}=-\sum_{t=1}^{N} \nabla[\phi(\triangle u)] u^{-}=\sum_{t=1}^{N-1} \phi(\triangle u) \triangle\left(u^{-}\right) \\
& =-\sum_{t=1}^{N-1} \phi\left(\triangle\left(u^{-}\right)\right) \triangle\left(u^{-}\right) \leq 0
\end{aligned}
$$

and hence $\triangle\left(\mathbf{u}^{-}\right)=0$. As $u(1)^{-}=u(N)^{-}=0$, we conclude that $\mathbf{u}^{-}=0$. Therefore $\mathbf{u}$ is a positive solution of (1.1) if and only if it is a nontrivial solution of (3.1).

We set

$$
D=\left\{\mathbf{u} \in Y:|\triangle \mathbf{u}|_{\infty}<1\right\}
$$

and denote $K: \mathbb{R}^{N} \rightarrow Y$ that sends any vector $\mathbf{v}$ onto the unique solution $\mathbf{w} \in Y$ of

$$
\left\{\begin{array}{l}
-\nabla[\phi(\Delta w(t))]=v(t), \quad t \in \mathbb{T} \\
w(1)=w(N)=0 .
\end{array}\right.
$$

The operator $N_{\lambda, \mu}: \bar{D} \rightarrow \mathbb{R}^{N}$ is defined by

$$
N_{\lambda, \mu}(u)=\bar{f}(\cdot, u)
$$

and the operator $F_{\lambda, \mu}=K \circ N_{\lambda, \mu}$ is completely continuous because $Y$ is a finite dimensional space. Obviously, a function $\mathbf{u}$ is a positive solution of (3.1) if and only if $\mathbf{u} \in D$ is a nontrivial fixed point of $F_{\lambda, \mu}$.

Step 2. Proof of (i). Take $\mu=0$ and fix $\lambda>0$. Assume (H) and (A1)-(A2). We further use the symbols $N$ and $F$ to replace the operators $N_{\lambda, 0}$ and $F_{\lambda, 0}$. Set $\Lambda_{a}=\lambda|\bar{a}|_{\infty}$ and let $\delta_{a} \in(0,1)$ be the constant $\delta_{a}=\delta$ introduced in Lemma 2.3, with $\Lambda=\Lambda_{a}$.

Let us consider the Dirichlet problem

$$
\left\{\begin{array}{l}
-\nabla[\phi(\triangle u(t))]=\lambda a_{1} u^{p_{1}}, \quad t \in \mathbb{T}_{1} \\
u\left(t_{1}\right)=u\left(t_{2}\right)=0
\end{array}\right.
$$

where $t_{1}, t_{2} \in \mathbb{R}, \mathbb{T}_{1}, a_{1}$ and $p_{1}$ are defined in (A1). Suppose that $\hat{\mathbb{T}}_{1} \subset \mathbb{T}$. By Lemma 2.6 there exists a solution $\boldsymbol{\alpha}$ satisfying $|\boldsymbol{\alpha}|_{\infty} \leq \frac{N-1}{2}$ and $\boldsymbol{\alpha} \gg \mathbf{0}$. Let us extend $\boldsymbol{\alpha}$ to a function $\tilde{\boldsymbol{\alpha}}$ satisfying $|\triangle \tilde{\boldsymbol{\alpha}}|_{\infty} \leq 1$ and

$$
-\frac{N-1}{2}<\tilde{\alpha}(t)<0, \quad t \in \hat{\mathbb{T}} \backslash \hat{\mathbb{T}}_{1}
$$

We define the open bounded subset of $Y$

$$
\mathrm{U}_{0}=\left\{\mathbf{u} \in Y: \mathbf{u} \gg \tilde{\boldsymbol{\alpha}},|\triangle \mathbf{u}|_{\infty}<1-\delta_{a}\right\},
$$


and $\mathbf{v}_{0} \in \mathbb{R}^{N}$ by setting

$$
\nu_{0}(t)=\lambda \bar{a}(t, \tilde{\alpha}(t)), \quad t \in \mathbb{T} .
$$

Note that $\mathbf{v}_{0} \geq \mathbf{0}$ in $\mathbb{T}, \mathbf{v}_{0}>\mathbf{0}$ in $\mathbb{T}_{1}$ and $\left|\mathbf{v}_{0}\right|_{\infty} \leq \Lambda_{a}$. Let $\mathbf{z}_{0}$ be the solution of the problem

$$
\left\{\begin{array}{l}
-\nabla[\phi(\Delta u(t))]=v_{0}(t), \quad t \in \mathbb{T}, \\
u(1)=u(N)=0 .
\end{array}\right.
$$

By Lemma 2.4, $\mathbf{z}_{0} \gg 0$ in $\hat{\mathbb{T}}$.

Claim $F$ has no fixed points on $\partial \mathrm{U}_{0}$ and $\operatorname{deg}\left(I-F, \mathrm{U}_{0}, 0\right)=1$.

We first prove that

$$
\operatorname{deg}\left(I-\mathbf{z}_{0}, \mathrm{U}_{0}, 0\right)=1
$$

It suffices to verify that $\mathbf{z}_{0} \in \mathrm{U}_{0}$. The condition $\left|\Delta \mathbf{z}_{0}\right|_{\infty}<1-\delta_{a}$ is satisfied by the definition of $\delta_{a}$. It remains to prove that $\mathbf{z}_{0} \geq \tilde{\boldsymbol{\alpha}}, t \in \mathbb{T}$. Since $z_{0}(t) \geq 0, t \in \mathbb{T}$ and $\tilde{\alpha}(t)<0, t \in \hat{\mathbb{T}} \backslash \hat{\mathbb{T}}_{1}$, we only need to show that $z_{0}(t)>\alpha(t), t \in \hat{\mathbb{T}}_{1}$. Since $z_{0}(t) \gg 0, t \in \hat{\mathbb{T}}$, we have $\min _{t \in \hat{\mathbb{1}}_{1}} z_{0}(t)>0$. Moreover, as $\lambda a_{1} u^{p_{1}} \leq v_{0}, t \in \mathbb{T}$ by (A1), we get, by Lemma 2.1,

$$
\alpha(t) \leq z_{0}(t)-\min _{\left\{t_{1}, t_{2}\right\}} z_{0}(t)<z_{0}(t)
$$

for all $t \in \hat{\mathbb{T}}_{1}$.

Next we consider the homotopy $H:[0,1] \times \bar{D} \rightarrow Y$ defined by

$$
H(k, u)=K\left(k N(u)+(1-k) v_{0}\right) .
$$

Obviously, $H$ is completely continuous. Note that

$$
H(0, u)=z_{0}, \quad H(1, u)=F(u)
$$

for all $\mathbf{u} \in \bar{D}$. Fix now $k \in[0,1]$ and suppose that $\mathbf{u} \in \overline{\mathrm{U}}_{0}$ is a fixed point of $H(k, \cdot)$. We will prove that $\mathbf{u} \in \mathrm{U}_{0}$. Since $\mathbf{u}$ is a fixed point of $H(k, \cdot)$, $\mathbf{u}$ is a solution of

$$
\left\{\begin{array}{l}
-\nabla[\phi(\Delta u(t))]=k \lambda a(t, u(t))+(1-k) v_{0}(t), \quad t \in \mathbb{T}, \\
u(1)=u(N)=0 .
\end{array}\right.
$$

Observe that

$$
k \lambda \bar{a}(t, x)+(1-k) v_{0}(t) \geq 0, \quad t \in \mathbb{T}, x \in\left[-\frac{N-1}{2}, 0\right] .
$$

Arguing as in Step 1, we see that $\mathbf{u} \geq \mathbf{0}$ in $\mathbb{T}$. Moreover, as

$$
k \lambda \bar{a}(t, u(t))+(1-k) v_{0}(t)>0, \quad t \in \mathbb{T}_{1},
$$


by Lemma 2.5 we deduce that $\min _{t \in \hat{T}_{1}} \mathbf{u}>0$. Let us prove that $\mathbf{u} \gg \tilde{\boldsymbol{\alpha}}$ in $\hat{\mathbb{T}}$. As above we observe that, since $u(t) \geq 0, t \in \mathbb{T}$ and $\tilde{\alpha}(t)<0, t \in \hat{\mathbb{T}} \backslash \hat{\mathbb{T}}_{1}$, we only need to show that $u(t)>\alpha(t), t \in \hat{\mathbb{T}}_{1}$. Note that, using (A1) and $\mathbf{u} \in \overline{\mathrm{U}}_{0}$, we have

$$
k \lambda \bar{a}(\cdot, u(t))+(1-k) v_{0}(t) \geq k \lambda a_{1} u^{p_{1}}+(1-k) \lambda \bar{a}(\cdot, \alpha(t)) \geq \lambda a_{1} \alpha^{p_{1}}, \quad t \in \mathbb{T}_{1} .
$$

Applying Lemma 2.1, we get

$$
\alpha(t) \leq u(t)-\min _{\left\{t_{1}, t_{2}\right\}} u<u(t)
$$

for all $t \in \hat{\mathbb{T}}$.

Furthermore, as

$$
\left|k \lambda \bar{a}(t, u(t))+(1-k) v_{0}(t)\right|_{\infty} \leq \Lambda_{a}
$$

Lemma 2.3 yields

$$
|\triangle \mathbf{u}|_{\infty}<1-\delta_{a}
$$

In conclusion, $\mathbf{u} \in \mathrm{U}_{0}$. The homotopy invariance of the degree implies that

$$
\operatorname{deg}\left(I-F, \mathrm{U}_{0}, 0\right)=\operatorname{deg}\left(I-\mathbf{z}_{0}, \mathrm{U}_{0}, 0\right)=1 .
$$

This concludes the proof of the claim.

Therefore, for every $\lambda>0$, there exists a nontrivial fixed point $\mathbf{u} \in \mathrm{U}_{0}$ of the operator $F$, i.e., there exists a positive solution $\mathbf{u}$ of (3.1) satisfying $\mathbf{u} \gg \tilde{\boldsymbol{\alpha}}$ in $\hat{\mathbb{T}}$.

Step 3. Proof of (ii). Take $\lambda=0$ and $\mu>0$. Assume (H) and (B1)-(B2). We further use the symbols $N$ and $F$ to replace the operators $N_{0, \mu}$ and $F_{0, \mu}$. Set $\Lambda_{b}=\mu|\bar{b}|_{\infty}$ and let $\delta_{b} \in(0,1)$ be the constant $\delta_{b}=\delta$ introduced in Lemma 2.3, with $\Lambda=\Lambda_{b}$.

Let us consider the Dirichlet problem

$$
\left\{\begin{array}{l}
-\nabla[\phi(\Delta u(t))]=\mu b_{1} u^{q_{1}}, \quad t \in \mathbb{T}_{1}, \\
u\left(t_{1}\right)=u\left(t_{2}\right)=0
\end{array}\right.
$$

where $b_{1}$ and $q_{1}$ are defined in (B1). We still suppose that $\hat{\mathbb{T}}_{1} \subset \mathbb{T}$. By Lemma 2.6 , there exists a constant $\mu^{*}>0$ such that, for any $\mu>\mu^{*}$, problem (3.3) has at least one solution $\boldsymbol{\alpha}_{1}$ satisfying $\left|\boldsymbol{\alpha}_{1}\right|_{\infty} \leq \frac{N-1}{2}$ and $\boldsymbol{\alpha}_{1} \gg \mathbf{0}$ in $\hat{\mathbb{T}}$. As in step 2 , we extend $\boldsymbol{\alpha}_{1}$ to a function $\tilde{\boldsymbol{\alpha}}_{1}$ satisfying $\left|\triangle \tilde{\boldsymbol{\alpha}}_{1}\right|_{\infty}<1$ and

$$
-\frac{N-1}{2}<\tilde{\boldsymbol{\alpha}}_{1}(t)<0, \quad t \in \hat{\mathbb{T}} \backslash \hat{\mathbb{T}}_{1} .
$$

We define the open bounded set

$$
\mathrm{U}_{1}=\left\{\mathbf{u} \in Y: \mathbf{u} \gg \boldsymbol{\alpha}_{1},|\triangle \mathbf{u}|_{\infty}<1-\delta_{b}\right\}
$$


and $\mathbf{v}_{1} \in \mathbb{R}^{N}$ by setting

$$
v_{1}(t)=\mu \bar{b}\left(t, \tilde{\alpha}_{1}(t)\right)
$$

The proof continues as in step 2, by showing that $F$ has no fixed points on $\partial \mathrm{U}_{1}$ and

$$
\operatorname{deg}\left(I-F, \mathrm{U}_{1}, 0\right)=1 .
$$

Therefore we conclude that, for all $\mu>\mu^{*}$, there exists a nontrivial fixed point $\mathbf{u} \in \mathrm{U}_{1}$ of $F$, i.e., there exists a positive solution $\mathbf{u}$ of (3.1) satisfying $\mathbf{u} \gg \tilde{\boldsymbol{\alpha}}_{1}$.

Step 4. Proof of (iii). Take $\lambda=0$ and $\mu>0$. Assume (H) and (B1)-(B3). Note that (B1) and (B3) together imply $q_{1}>1$. Similarly, we use the symbols $N$ and $F$ to denote the operators $N_{0, \mu}$ and $F_{0, \mu}$. Let $\mu^{*}$ be the constant, whose existence was proved in Step 3, such that problem (3.1) has at least one positive solution for all $\mu>\mu^{*}$. Fix $\mu>\mu^{*}$ and let $\mathbf{u}_{1} \in \mathrm{U}_{1}$ be a corresponding solution. Let us prove the existence of a second positive solution.

For each $r>0$, we set

$$
\mathrm{U}_{2}^{r}=\left\{\mathbf{u} \in Y:|\mathbf{u}|_{\infty}<r,|\triangle \mathbf{u}|_{\infty}<1-\delta_{b}\right\}
$$

with $\delta_{b}$ defined in Step 3.

Claim There exists $\hat{r}>0$ such that, for each $r \in(0, \hat{r}], F$ has no fixed points on $\partial \mathrm{U}_{2}^{r}$ and

$$
\operatorname{deg}\left(I-F, \mathrm{U}_{2}^{r}, 0\right)=1 \text {. }
$$

Consider the homotopy $H:[0,1] \times \bar{D} \rightarrow Y$ defined by

$$
H(k, u)=K(k N(u)) .
$$

Obviously, $H$ is completely continuous. We have

$$
H(0, u)=0, \quad H(1, u)=F(u)
$$

for all $\mathbf{u} \in \bar{D}$. Fix $k \in[0,1]$ and suppose that $\mathbf{u} \in \overline{\mathrm{U}}_{2}^{r}$ is a fixed point of $H(k, \cdot)$. We will prove that $\mathbf{u} \in \mathrm{U}_{2}^{r}$. Since $\mathbf{u}$ is a fixed point of $H(k, \cdot), \mathbf{u}$ is a solution of

$$
\left\{\begin{array}{l}
-\nabla[\phi(\triangle u(t))]=k \mu \bar{b}(t, u(t)), \quad t \in \mathbb{T}, \\
u(1)=u(N)=0,
\end{array}\right.
$$

multiplying the equation in (3.4) by $u(t)$ and summing, we obtain

$$
\begin{aligned}
\sum_{t=2}^{N-1}(\Delta u(t))^{2} & \leq \sum_{t=2}^{N-1} \phi(\Delta u(t)) \Delta u(t)=\sum_{t=2}^{N-1} k \mu \bar{b}(t, u(t)) u(t) \\
& \leq \mu b_{2} \sum_{t=2}^{N-1}|u|^{q_{2}+1} \leq \mu b_{2} r^{q_{2}-1} \sum_{t=2}^{N-1} u^{2} \\
& \leq \mu b_{2} r^{q_{2}-1} \frac{1}{\lambda_{1} \min _{t \in \mathbb{T}} m(t)} \sum_{t=2}^{N-1}(\Delta u(t))^{2},
\end{aligned}
$$


where $\lambda_{1}$ is the smallest eigenvalue of (2.8). Hence there exists a sufficiently small $\hat{r}>0$ such that, for every $r \in(0, \hat{r}]$, we have $\Delta \mathbf{u}=0$, and therefore $\mathbf{u}=\mathbf{0}$. The homotopy invariance of the degree implies that

$$
\operatorname{deg}\left(I-F, \mathrm{U}_{2}^{r}, 0\right)=1
$$

This concludes the proof of the claim.

We finally set

$$
\mathrm{U}_{3}=\left\{\mathbf{u} \in Y:|\triangle \mathbf{u}|_{\infty}<1-\delta_{b}\right\} .
$$

Using the definition of $\delta_{b}$ and arguing as above, we easily see that

$$
\operatorname{deg}\left(I-F, \mathrm{U}_{3}, 0\right)=1 .
$$

Let us fix $r \in\left(0, \min \left\{\left|\tilde{\boldsymbol{\alpha}}_{1}\right|_{\infty}, \hat{r}\right\}\right]$, with $\tilde{\boldsymbol{\alpha}}_{1}$ defined in Step 3. Notice that the sets $\mathrm{U}_{1}$ and $\mathrm{U}_{2}^{r}$, previously defined, are disjoint and both contained in $\mathrm{U}_{3}$. Let us define

$$
\mathrm{W}^{r}=\mathrm{U}_{3} \backslash\left(\overline{\mathrm{U}_{1} \cup \mathrm{U}_{2}^{r}}\right) .
$$

As $F$ has no fixed point in $\partial \mathrm{U}_{1} \cup \partial \mathrm{U}_{2}^{r} \cup \partial \mathrm{U}_{3}$, by the excision and additivity properties of the degree, we have

$$
\begin{aligned}
\operatorname{deg}\left(I-F, \mathrm{U}_{3}, 0\right) & =\operatorname{deg}\left(I-F, \mathrm{U}_{3} \backslash\left(\partial \mathrm{U}_{1} \cup \partial \mathrm{U}_{2}^{r}\right), 0\right) \\
& =\operatorname{deg}\left(I-F, \mathrm{U}_{1}, 0\right)+\operatorname{deg}\left(I-F, \mathrm{U}_{2}^{r}, 0\right)+\operatorname{deg}\left(I-F, \mathrm{~W}^{r}, 0\right)
\end{aligned}
$$

and hence

$$
\operatorname{deg}\left(I-F, \mathrm{~W}^{r}, 0\right)=-1
$$

In particular, there exists a fixed point $\mathbf{u}_{2}$ of $F$ such that $\left|\mathbf{u}_{2}\right|_{\infty}>r$ and for which the condition $\mathbf{u}_{2} \gg \boldsymbol{\alpha}_{1}$ does not hold. Therefore $\mathbf{u}_{2}$ is a positive solution of (3.1) which differs from $\mathbf{u}_{1}$. We conclude that, for all $\mu>\mu^{*}$, there exist at least two positive solutions of (3.1).

Step 5. Proof of (iv). Take $\lambda>0$ and $\mu>0$. Assume (H), (A1)-(A3) and (B1)-(B3). Again, we can suppose that $\hat{\mathbb{T}}_{1} \subset \mathbb{T}$. As already noticed in Step 4 , we have $q_{1}>1$. Let $\mu^{*}$ be the constant, introduced in Step 3, such that problem (3.1), with $\lambda=0$, has at least one positive solution for all $\mu>\mu^{*}$. Fix $\mu>\mu^{*}$, set

$$
\Lambda=|\bar{a}|_{\infty}+\mu|\bar{b}|_{\infty}
$$

and let $\delta \in(0,1)$ be the constant introduced in Lemma 2.3. Let us take $\hat{\mathbb{T}}_{2} \subset \mathbb{T}_{1}$, and consider, for $\lambda \in(0,1]$, the Dirichlet problem

$$
\left\{\begin{array}{l}
-\nabla[\phi(\Delta u(t))]=\lambda a_{1} u^{p_{1}}, \quad t \in \mathbb{T}_{2}, \\
u\left(t_{3}\right)=u\left(t_{4}\right)=0
\end{array}\right.
$$


where $t_{3}, t_{4} \in \mathbb{R}$. By Lemma 2.6 there exists a solution $\boldsymbol{\alpha}_{2}^{\lambda}$ of (3.5) satisfying $\left|\boldsymbol{\alpha}_{2}^{\lambda}\right|_{\infty} \leq \frac{N-1}{2}$ and $\boldsymbol{\alpha}_{2}^{\lambda} \gg \mathbf{0}$.

Denote by $c_{0}>0$ a constant, dependent on $\Lambda$ and $\mathbb{T}_{2}$, such that

$$
|\mathbf{u}|_{\infty} \leq c_{0}|\mathbf{v}|_{\infty}
$$

holds for all $\mathbf{v} \in \mathbb{R}^{N}$ satisfying $|\mathbf{v}|_{\infty} \leq \Lambda$. Similarly, denote by $c_{0}^{\prime}$ a constant, dependent on $\Lambda, \mathbb{T}$, such that

$$
|\mathbf{u}|_{\infty} \leq c_{0}^{\prime}|\mathbf{v}|_{\infty}
$$

holds for all $\mathbf{v} \in \mathbb{R}^{N}$ satisfying $|\mathbf{v}|_{\infty} \leq \Lambda$. Set $c_{1}=\Lambda \max \left\{c_{0}, c_{0}^{\prime}\right\}$ and $r_{\lambda}=\lambda\left(c_{1}+1\right)$. Observe that, since by (A1), $\left|a_{1}\left(\boldsymbol{\alpha}_{2}^{\lambda}\right)^{p_{1}}\right|_{\infty} \leq \Lambda$, we have

$$
\left|\boldsymbol{\alpha}_{2}^{\lambda}\right|_{\infty} \leq c_{0} \Lambda \lambda \leq c_{1} \lambda
$$

As in Step 3, let $\boldsymbol{\alpha}_{1}$ be a solution of the Dirichlet problem

$$
\left\{\begin{array}{l}
-\nabla[\phi(\Delta u(t))]=\mu b_{1} u^{q_{1}}, \quad t \in \mathbb{T}_{1}, \\
u\left(t_{1}\right)=u\left(t_{2}\right)=0 .
\end{array}\right.
$$

Since $\boldsymbol{\alpha}_{1} \gg \mathbf{0}, t \in \hat{\mathbb{T}}$, we have $\min _{t \in \hat{\mathbb{T}}_{2}} \boldsymbol{\alpha}_{1}>\mathbf{0}$. Therefore we can take $\bar{\lambda} \in(0,1]$ such that, for all $\lambda \in(0, \bar{\lambda}), r_{\lambda}<\min _{t \in \hat{\mathbb{T}}_{2}} \boldsymbol{\alpha}_{1}$. For all $\lambda \in(0, \bar{\lambda})$, we extend $\boldsymbol{\alpha}_{1}$ to a function $\tilde{\boldsymbol{\alpha}}_{1}^{\lambda}$ and $\boldsymbol{\alpha}_{2}^{\lambda}$ to a function $\tilde{\boldsymbol{\alpha}}_{2}^{\lambda}$ such that

$$
\begin{aligned}
& \left|\triangle \tilde{\boldsymbol{\alpha}}_{1}^{\lambda}\right|_{\infty} \leq 1, \quad\left|\triangle \tilde{\boldsymbol{\alpha}}_{2}^{\lambda}\right|_{\infty} \leq 1, \quad\left|\triangle \tilde{\boldsymbol{\alpha}}_{2}^{\lambda}\right|_{\infty} \leq r_{\lambda}, \\
& -\frac{N-1}{2}<\tilde{\alpha}_{2}^{\lambda}(t)<\tilde{\alpha}_{1}^{\lambda}(t)<0, \quad t \in \hat{\mathbb{T}} \backslash \hat{\mathbb{T}}_{1}, \\
& -\frac{N-1}{2}<\tilde{\alpha}_{2}^{\lambda}(t)<0, \quad t \in \hat{\mathbb{T}}_{1} \backslash \hat{\mathbb{T}}_{2} .
\end{aligned}
$$

We define, for every $\lambda \in(0, \bar{\lambda})$, the open bounded sets

$$
\mathrm{V}_{1}^{\lambda}=\left\{\mathbf{u} \in Y: \mathbf{u} \gg \tilde{\boldsymbol{\alpha}}_{1}^{\lambda},|\triangle \mathbf{u}|_{\infty}<1-\delta\right\}
$$

and

$$
\mathrm{V}_{2}^{\lambda}=\left\{\mathbf{u} \in Y: \mathbf{u} \gg \tilde{\boldsymbol{\alpha}}_{2}^{\lambda},|\mathbf{u}|_{\infty}<r_{\lambda},|\Delta \mathbf{u}|_{\infty}<1-\delta\right\}
$$

We also set, for $t \in \mathbb{T}$,

$$
v_{1}^{\lambda}(t)=\mu \bar{b}\left(t, \tilde{\alpha}_{1}^{\lambda}(t)\right)
$$

and

$$
v_{2}^{\lambda}(t)=\lambda \bar{a}\left(t, \tilde{\alpha}_{2}^{\lambda}(t)\right) .
$$


For every $\lambda \in(0, \bar{\lambda})$, let $\mathbf{z}_{1}^{\lambda}$ be the solution of the Dirichlet problem

$$
\left\{\begin{array}{l}
-\nabla[\phi(\Delta u(t))]=v_{1}^{\lambda}(t), \quad t \in \mathbb{T} \\
u(1)=u(N)=0 .
\end{array}\right.
$$

Arguing as in the claim of Step 2, we easily verify that $F_{\lambda, \mu}$ has no fixed points on $\partial V_{1}^{\lambda}$ and

$$
\operatorname{deg}\left(I-F_{\lambda, \mu}, V_{1}^{\lambda}, 0\right)=1
$$

For every $\lambda \in(0, \bar{\lambda})$, let $\mathbf{z}_{2}^{\lambda}$ be the solution of the Dirichlet problem

$$
\left\{\begin{array}{l}
-\nabla[\phi(\Delta u(t))]=v_{2}^{\lambda}(t), \quad t \in \mathbb{T}, \\
u(1)=u(N)=0 .
\end{array}\right.
$$

Claim There exists $\lambda(\mu) \in(0, \bar{\lambda}]$ such that, for all $\lambda \in(0, \lambda(\mu)), F_{\lambda, \mu}$ has no fixed points on $\partial V_{2}^{\lambda}$ and

$$
\operatorname{deg}\left(I-F_{\lambda, \mu}, \mathrm{V}_{2}^{\lambda}, 0\right)=1
$$

We first prove that

$$
\operatorname{deg}\left(I-\mathbf{z}_{2}^{\lambda}, \mathrm{V}_{2}^{\lambda}, 0\right)=1
$$

It suffices to verify that $\mathbf{z}_{2}^{\lambda} \in \mathrm{V}_{2}^{\lambda}$. Arguing as in Step 2, we easily see that $\left|\triangle \mathbf{z}_{2}^{\lambda}\right|_{\infty}<1-\delta$ and $\mathbf{z}_{2}^{\lambda} \gg \boldsymbol{\alpha}_{2}^{\lambda}$ in $\mathbb{T}$. Furthermore, we have

$$
\left|\mathbf{z}_{2}^{\lambda}\right|_{\infty} \leq c_{0}^{\prime} \Lambda \lambda \leq c_{1} \lambda<r_{\lambda}
$$

Next we consider the homotopy $H:[0,1] \times \bar{D} \rightarrow Y$ defined by

$$
H(k, u)=K\left(k N_{\lambda, \mu}(u)+(1-k) v_{2}^{\lambda}\right) .
$$

Obviously, $H$ is completely continuous. Observe that

$$
H(0, u)=z_{2}^{\lambda}, \quad H(1, u)=F_{\lambda, \mu}(u)
$$

for all $\mathbf{u} \in \bar{D}$.

Fix now $k \in[0,1]$, and suppose that $\mathbf{u} \in \overline{\mathrm{V}}_{2}^{\lambda}$ is a fixed point of $H(k, \cdot)$. We will prove that $\mathbf{u} \in \mathrm{V}_{2}^{\lambda}$. Arguing as in step 2 we easily show that $\boldsymbol{u} \geq \mathbf{0}$ in $\mathbb{T}$ and $\min _{\widehat{\mathbb{T}}_{2}} \mathbf{u}>0$. Let us prove that $\mathbf{u} \gg \tilde{\boldsymbol{\alpha}}_{2}^{\lambda}$ in $\mathbb{T}$. Since $u(t) \geq 0, t \in \mathbb{T}$ and $\tilde{\alpha}_{2}^{\lambda}(t)<0, t \in \hat{\mathbb{T}} \backslash \hat{\mathbb{T}}_{2}$, we only need to verify that $u(t)>\alpha_{2}^{\lambda}(t), t \in \hat{\mathbb{T}}_{2}$. Note that

$$
k \bar{f}(\cdot, u)+(1-k) v_{2}^{\lambda} \geq k \lambda a_{1} u^{p_{1}}+(1-k) \lambda \bar{a}\left(\cdot, \alpha_{2}^{\lambda}\right) \geq \lambda a_{1}\left(\alpha_{2}^{\lambda}\right)^{p_{1}}, \quad t \in \mathbb{T} .
$$

Applying Lemma 2.1, we get

$$
\alpha_{2}^{\lambda}(t) \leq u(t)-\min _{\left\{t_{3}, t_{4}\right\}} u<u(t), \quad t \in \hat{\mathbb{T}}_{2} .
$$


Moreover, as

$$
\left|k \bar{f}(\cdot, u)+(1-k) v_{2}^{\lambda}\right|_{\infty} \leq \Lambda
$$

Lemma 2.3 yields

$$
|\triangle \mathbf{u}|_{\infty}<1-\delta
$$

Finally, we verify that $|\mathbf{u}|_{\infty}<r_{\lambda}$ if $\lambda$ is sufficiently small. Since both $|\mathbf{u}|_{\infty} \leq r_{\lambda}$ and $\left|\boldsymbol{\alpha}_{2}^{\lambda}\right|_{\infty} \leq$ $r_{\lambda}$ hold, we have

$$
\begin{aligned}
k \bar{f}(\cdot, u)+(1-k) v_{2}^{\lambda} & \leq k\left(\lambda a_{2}|u|_{\infty}^{p_{2}}+\mu b_{2}|u|_{\infty}^{q_{2}}\right)+(1-k) \lambda a_{2}\left|\tilde{\alpha}_{2}^{\lambda}\right|_{\infty}^{p_{2}} \\
& \leq k\left(a_{2}\left(c_{1}+1\right)^{p_{2}} \lambda^{p_{2}+1}+\mu b_{2}\left(c_{1}+1\right)^{q_{2}} \lambda^{q_{2}}+(1-k) a_{2}\left(c_{1}+1\right)^{p_{2}} \lambda^{p_{2}+1}\right. \\
& \leq a_{2}\left(c_{1}+1\right)^{p_{2}} \lambda^{p_{2}+1}+\mu b_{2}\left(c_{1}+1\right)^{q_{2}} \lambda^{q_{2}} \leq c_{2} \lambda^{1+\epsilon}, \quad t \in \mathbb{T},
\end{aligned}
$$

where $c_{2}>0$ is a constant independent of $\lambda$ and $\epsilon=\min \left\{p_{2}, q_{2}-1\right\}$. Applying Lemma 2.3, we obtain

$$
|\mathbf{u}|_{\infty} \leq c_{3} \lambda^{1+\epsilon}
$$

where $c_{3}>0$ is a constant independent of $\lambda$. Let $\lambda(\mu) \in(0, \bar{\lambda})$ be such that

$$
\lambda(\mu) \leq\left(\frac{c_{1}+1}{c_{3}}\right)^{\frac{1}{\epsilon}}
$$

Then, for each $\lambda \in(0, \lambda(\mu))$, the inequality $|\mathbf{u}|_{\infty}<r_{\lambda}$ holds and hence $\mathbf{u} \in \mathrm{V}_{2}^{\lambda}$. The homotopy invariance of the degree implies then that

$$
\operatorname{deg}\left(I-F_{\lambda, \mu}, \mathrm{V}_{2}^{\lambda}, 0\right)=1
$$

This concludes the proof the claim.

Note that $V_{1}^{\lambda}$ and $V_{2}^{\lambda}$ are disjoint because of the choice of $\lambda$. Therefore problem (3.1) has at least two positive solutions $\mathbf{u}_{1}$ and $\mathbf{u}_{2}$ such that $\mathbf{u}_{1} \gg \boldsymbol{\alpha}_{1}^{\lambda}$ and $\left|\mathbf{u}_{2}\right|_{\infty}<r_{\lambda}$. Finally we define, for all $\lambda \in(0, \lambda(\mu))$,

$$
\mathrm{V}_{3}^{\lambda}=\left\{\mathbf{u} \in Y: \mathbf{u}_{1} \gg \tilde{\boldsymbol{\alpha}}_{2}^{\lambda},|\triangle \mathbf{u}|_{\infty}<1-\delta\right\}
$$

We also set $\mathrm{W}^{\lambda}=\mathrm{V}_{3}^{\lambda} \backslash\left(\overline{\mathrm{V}_{1}^{\lambda} \cup V_{2}^{\lambda}}\right)$. Fix $\lambda \in(0, \lambda(\mu))$. Arguing as in the first part of the previous claim, we easily verify that

$$
\operatorname{deg}\left(I-F_{\lambda, \mu}, \mathrm{V}_{3}^{\lambda}, 0\right)=1
$$

By the excision and the additivity properties of the degree, we obtain

$$
\operatorname{deg}\left(I-F_{\lambda, \mu}, \mathrm{W}^{\lambda}, 0\right)=-1
$$


Hence, there exists a fixed point $\mathbf{u}_{3}$ of $F_{\lambda, \mu}$ such that $\left|\mathbf{u}_{3}\right|_{\infty}>r_{\lambda}$ and for which the condition $\mathbf{u}_{3} \gg \tilde{\boldsymbol{\alpha}}_{1}^{\lambda}$ in $\hat{\mathbb{T}}$ does not hold. Therefore $\mathbf{u}_{3}$ is a positive solution of (3.1) which differs both from $\mathbf{u}_{1}$ and from $\mathbf{u}_{2}$. We conclude that, for every $\mu>\mu^{*}, \lambda \in(0, \lambda(\mu))$, problem (3.1) has at least three positive solutions.

Finally, we give an example to illustrate the conclusion of Theorem 3.1.

Consider the Dirichlet problem

$$
\left\{\begin{array}{l}
-\nabla[\phi(\Delta u(t))]=\frac{3}{2} \lambda u^{\frac{1}{2}}+2 \mu u^{2}, \quad t \in \mathbb{T}, \\
u(1)=u(N)=0 .
\end{array}\right.
$$

In this case, $a(t, u)=\frac{3}{2} u^{\frac{1}{2}}, b(t, u)=2 u^{2}$.

Take $a_{1}=1, p_{1}=\frac{1}{2}$. It is easy to check that the assumptions of (i) of Theorem 3.1 are satisfied. Hence, when $\mu=0$, for every $\lambda>0$, problem (3.6) has at least one positive solution.

Take $b_{1}=1, p_{1}=2$. It is easy to check that the assumptions of (ii) of Theorem 3.1 are satisfied. Hence, when $\lambda=0$, there exists $\mu^{*}>0$ such that, for every $\mu>\mu^{*}$, problem (3.6) has at least one positive solution.

Take $b_{2}=3, p_{2}=2$. It is easy to check that the assumptions of (iii) of Theorem 3.1 are satisfied. Hence, when $\lambda=0$, then there exists $\mu^{*}>0$ such that, for every $\mu>\mu^{*}$, problem (3.6) has at least two positive solutions.

Take $a_{2}=2, p_{2}=\frac{1}{2}$. It is easy to check that all the assumptions of (iv) of Theorem 3.1 are satisfied. Then there exist $\mu^{*}>0$ and a function $\lambda(\cdot):\left(\mu^{*},+\infty\right) \rightarrow \mathbb{R}$ such that, for every $\mu>\mu^{*}, \lambda \in(0, \lambda(\mu))$, problem (3.6) has at least three positive solutions.

\author{
Acknowledgements \\ The authors would like to thank the editor and the anonymous referees for their helpful suggestions. \\ Funding \\ This work was supported by the National Natural Science Foundation of China (No. 11671322). \\ Abbreviations \\ Not applicable. \\ Availability of data and materials \\ Data sharing not applicable to this article as no datasets were generated. \\ Competing interests \\ The authors declare that they have no competing interests. \\ Authors' contributions \\ The authors claim that the research was realized in collaboration with the same responsibility. All authors read and \\ approved the last of the manuscript.
}

\title{
Publisher's Note
}

Springer Nature remains neutral with regard to jurisdictional claims in published maps and institutional affiliations.

Received: 23 July 2020 Accepted: 23 November 2020 Published online: 01 December 2020

\section{References}

1. Bereanu, C., Mawhin, J.: Existence and multiplicity results for some nonlinear problems with singular $\phi$-Laplacian. J. Differ. Equ. 43, 536-557 (2007)

2. Bereanu, C., Mawhin, J.: Boundary value problems for second-order nonlinear difference equations with discrete $\phi$-Laplacian and singular $\phi$. J. Differ. Equ. Appl. 14, 1099-1118 (2008)

3. Bereanu, C., Thompson, H.B.: Periodic solutions of second order nonlinear difference equations with discrete $\phi$-Laplacian. J. Math. Anal. Appl. 330, 1002-1015 (2007)

4. Cabada, A., Otero-Espinar, V.: Existence and comparison results for difference $\phi$-Laplacian boundary value problems with lower and upper solutions in reverse order. J. Math. Anal. Appl. 267, 501-521 (2002) 
5. Chen, T., Ma, R.: Solvability for some boundary value problems with discrete $\phi$-Laplacian operators. Adv. Differ. Equ. 2015, Article ID 139 (2015)

6. Coelho, I., Corsato, C., Obersnel, F., Omari, P.: Positive solutions of the Dirichlet problem for the one-dimensional Minkowski-curvature equation. Adv. Nonlinear Stud. 12, 621-638 (2012)

7. Corsato, C., Obersnel, F., Omari, P., Rivetti, S.: Positive solutions of the Dirichlet problem for the prescribed mean curvature equation in Minkowski space. J. Math. Anal. Appl. 405, 227-239 (2013)

8. Kelley, W.G., Peterson, A.C.: Difference Equations: An Introduction with Applications, 2nd edn. Harcourt/Academic Press, San Diego (2001)

9. Ma, R., Gao, C.: Spectrum of discrete second-order difference operator with sign-changing weight and its applications. Discrete Dyn. Nat. Soc. 2014, Article ID 590968 (2014)

10. Ma, R., Gao, C., Ma, H., Lu, Y.: Theory of Difference Equations and Applications. Science Press, Bei Jing (2019) (in Chinese)

11. Ma, R., Lu, Y.: Periodic solutions of second order nonlinear difference equations with singular $\phi$-Laplacian operator. Discrete Dyn. Nat. Soc. 2014, Article ID 637242 (2014)

12. Ma, R., Wei, L., Chen, Z:: Evolution of bifurcation curves for one-dimensional Minkowski-curvature problem. Appl. Math. Lett. 103, 1-8 (2020)

13. Mawhin, J.: Periodic solutions of second order nonlinear difference systems with $\phi$-Laplacian: a variational approach. Nonlinear Anal. 75, 4672-4687 (2012)

14. $\mathrm{Xu}, \mathrm{M} ., \mathrm{Ma}, \mathrm{R} ., \mathrm{He}, \mathrm{Z}$ :: Positive solutions of the periodic problems for quasilinear difference equation with sign-changing weight. Adv. Differ. Equ. 2018, Article ID 393 (2018)

\section{Submit your manuscript to a SpringerOpen ${ }^{\circ}$ journal and benefit from:}

- Convenient online submission

- Rigorous peer review

- Open access: articles freely available online

- High visibility within the field

- Retaining the copyright to your article

Submit your next manuscript at $\boldsymbol{\nabla}$ springeropen.com 